\title{
Anaphylaxie - es tut sich was!
}

A naphylaxien stellen die Maximalform allergischer Erkrankungen dar. Dennoch sind mögliche Erscheinungsformen und die Behandlung von Anaphylaxien wenig im Bewusstsein von Ärzten und Patienten verankert und rufen viel Verunsicherung hervor. Gerade bei Anaphylaxien ist jedoch schnelles und adäquates Handeln geboten, da hiervon die Überlebenschancen des Patienten maßgeblich abhängen.

Erfreulicherweise haben sich in letzter Zeit zahlreiche Arbeitsgruppen gebildet, die sich in verschiedenen Aktivitäten dem Thema Anaphylaxie widmen. Kurz vor der Vollendung befindet sich die unter der Leitung von J. Ring, München, erstellte Leitlinie zur Behandlung anaphylaktischer Reaktionen. Sie wird die Handlungsempfehlungen zur Anaphylaxiebehandlung aktualisieren und somit zur Verbesserung und Vereinheitlichung der therapeutischen Strategien beitragen.

Zwei andere Arbeitsgruppen widmen sich der Datensammlung. Unter der Leitung von M. Worm, Berlin wurde ein Anaphylaxieregister erstellt, in das bereits zahlreiche Kliniken in Deutschland, Österreich und der Schweiz ihre Anaphylaxiefälle eingegeben haben. Unter www.anaphylaxie.net sind auch Sie herzlich gebeten, sich regis-

„Ergebnis einer Patientenbefragung: Die sich an eine Anaphylaxie anschließende Aufklärung ist vielfach absolut unzureichend. Weniger als die Hälfte der Patienten wird einem Allergologen vorgestellt, und nur etwa $50 \%$ erhalten ein angemessenes Notfallset" trieren zu lassen und weitere Fallbeschreibungen beizusteuern.

Eine weitere wichtige und aktuelle Datensammlung verdanken wir einer Patientenbefragung unter den Mitgliedern des Deutschen Allergie- und Asthmabundes (DAAB). Wesentliche Erkenntnisse aus dieser Befragung waren, dass offensichtlich viele Patienten mehrfach Anaphylaxien erleiden, und dass die an das Erstereignis anschließende Aufklärung vielfach absolut unzureichend ist. Weniger als die Hälfte der Patienten wurden einem Allergologen vorgestellt, und nur

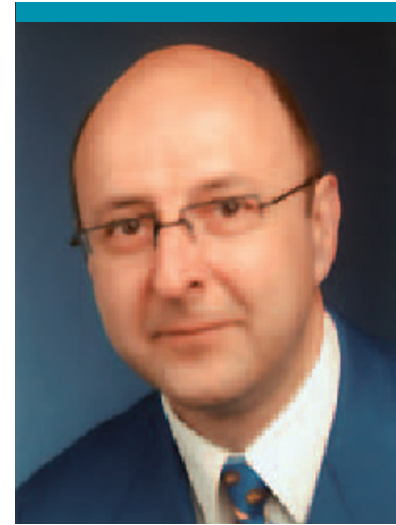

Prof. Dr. Wolfgang Czech, Praxis Dermatologie und Allergologie, VS-Villingen, Präsident des ÄDA

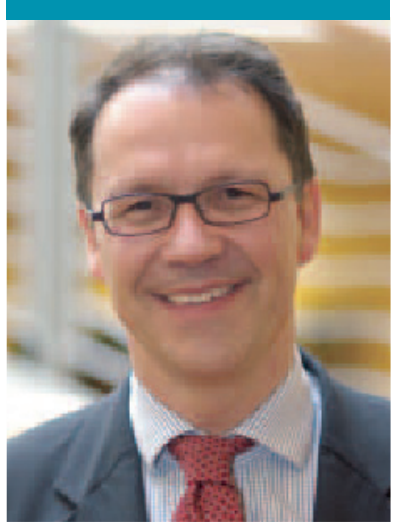

Prof. Dr. Ludger Klimek, Zentrum für Rhinologie und Allergologie, Wiesbaden, Vorstandsmitglied des ÄDA etwa 50\% erhielten ein angemessenes Notfallset.

Das „Expertenforum Anaphylaxie“ hat es sich in dieser Situation zum Ziel gesetzt, ein besseres Bewusstsein bei Ärzten und Öffentlichkeit zu schaffen. Zwei Broschüren - eine für Patienten, eine für Ärzte und medizinisches Fachpersonal - wurden erstellt und können über www.anaphylaxie-hilfe.de angefordert werden. Weitere Informationen sind auch über www.allergiezentrum.org zu erhalten.

Wir hoffen, dass das Thema Anaphylaxie somit eine breitere Beachtung in der medizinischen Öffentlichkeit findet und somit die Bedeutung von Allergien insgesamt dargestellt werden kann.

Wir wünschen Ihnen viel Freude beim Lesen dieses aktuellen Heftes des Allergo Journal,

Ihre
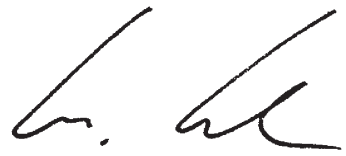

Prof. Dr. Wolfgang Czech<smiles>C=CC=CC</smiles>

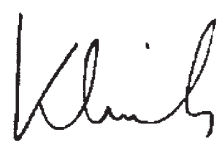

Prof. Dr. Ludger Klimek 